Bull. Korean Math. Soc. 48 (2011), No. 6, pp. 1169-1182

http://dx.doi.org/10.4134/BKMS.2011.48.6.1169

\title{
ON EXISTENCE OF WEAK SOLUTIONS OF NEUMANN PROBLEM FOR QUASILINEAR ELLIPTIC EQUATIONS INVOLVING $p$-LAPLACIAN IN AN UNBOUNDED DOMAIN
}

Trinh Thi Minh Hang and Hoang Quoc Toan

\begin{abstract}
In this paper we study the existence of non-trivial weak solutions of the Neumann problem for quasilinear elliptic equations in the form$$
-\operatorname{div}\left(h(x)|\nabla u|^{p-2} \nabla u\right)+b(x)|u|^{p-2} u=f(x, u), \quad p \geq 2
$$

in an unbounded domain $\Omega \subset \mathbb{R}^{N}, N \geq 3$, with sufficiently smooth bounded boundary $\partial \Omega$, where $h(x) \in L_{\text {loc }}^{1}(\bar{\Omega}), \bar{\Omega}=\Omega \cup \partial \Omega, h(x) \geq 1$ for all $x \in \Omega$. The proof of main results rely essentially on the arguments of variational method.
\end{abstract}

\section{Introduction and preliminaries results}

We are concerned with the study of a Neumann problem of the type

$$
\left\{\begin{array}{l}
-\operatorname{div}\left(h(x)|\nabla u|^{p-2} \nabla u\right)+b(x)|u|^{p-2} u=f(x, u) \quad \text { in } \Omega, \\
\frac{\partial u}{\partial n}=0 \quad \text { on } \partial \Omega, \quad u(x) \rightarrow 0 \text { as }|x| \rightarrow+\infty,
\end{array}\right.
$$

where $p \geq 2, \Omega \subset \mathbb{R}^{N}, N \geq 3$, is an unbounded domain with sufficiently smooth bounded boundary $\partial \Omega, \bar{\Omega}=\Omega \cup \partial \Omega, n$ is the outward unit normal to $\partial \Omega, f: \Omega \times \mathbb{R} \longrightarrow \mathbb{R}$ is a function which will be specified later, $h(x)$ and $b(x)$ are satisfied the following conditions:

(H) $h(x) \in L_{\text {loc }}^{1}(\bar{\Omega}), h(x) \geq 1$ for all $x \in \bar{\Omega}$.

(B) $b(x) \in L_{\mathrm{loc}}^{\infty}(\bar{\Omega}), b(x) \geq b_{0}>0$ for all $x \in \bar{\Omega}$.

We first make some comments on the problem (1.1). In the case when $\Omega$ is a bounded domain in $\mathbb{R}^{N}$ or $h(x)=1$ there were extensive studies in the last decades dealing with the Neumann problems of type (1.1). We just remember the papers $[1,2,4,3],[10,12,13,16]$, where different techniques of finding

Received June 11, 2010

2010 Mathematics Subject Classification. 35J20, 35 J65.

Key words and phrases. Neumann problem, p-Laplacian, Mountain pass theorem, the weakly continuously differentiable functional.

Research supported by the National Foundation for Science and Technology Development of Vietnam (NAFOSTED). 
solutions are illustrated. We also find that in the case that $h(x) \in L_{\text {loc }}^{1}(\Omega)$, the quasilinear elliptic equations of type (1.1), with Dirichlet boundary condition, have been studied by D. M. Duc, N. T. Vu ([7]), H. Q. Toan, N. Q. Anh, N. T. Chung (see $[15,14,5]$ ). The goal of this work we study the existence of weak solutions of Neumann problem for quasilinear elliptic equations with singular coefficients involving the $p$-Laplace operator of type (1.1) in an unbounded domain $\Omega \subset \mathbb{R}^{N}$ with sufficiently smooth bounded boundary $\partial \Omega$.

In order to state our main results let us introduce following some hypotheses:

(F1) $f(x, t) \in C^{1}(\Omega \times \mathbb{R}, \mathbb{R}), f(x, 0)=0, x \in \bar{\Omega}$.

(F2) There exist functions $\tau: \bar{\Omega} \longrightarrow \mathbb{R}, \tau(x) \geq 0$ for $x \in \bar{\Omega}$ and constant $r \in\left(p-1, \frac{N+p}{N-p}\right)$ such that

$$
\begin{aligned}
& \left|f_{z}^{\prime}(x, z)\right| \leq \tau(x)|z|^{r-1} \quad \text { for a.e. } x \in \bar{\Omega}, \\
& \tau(x) \in L^{\infty}(\Omega) \cap L^{r_{0}}(\Omega), \quad r_{0}=\frac{N p}{N p-(r+1)(N-p)} .
\end{aligned}
$$

(F3) There exists $\mu>p$ such that

$$
0<\mu F(x, z)=\mu \int_{0}^{z} f(x, t) d t \leq z f(x, z), x \in \bar{\Omega}, z \neq 0 .
$$

Denote by

$$
C_{0}^{\infty}(\bar{\Omega})=\left\{u \in C^{\infty}(\bar{\Omega}): \operatorname{supp} u \text { compact } \subset \bar{\Omega}\right\}
$$

and $W^{1, p}(\Omega)$ is the usual Sobolev space which can be defined as the completion of $C_{0}^{\infty}(\bar{\Omega})$ under the norm

$$
\|u\|=\left(\int_{\Omega}\left(|\nabla u|^{p}+|u|^{p}\right) d x\right)^{\frac{1}{p}} .
$$

We now consider following subspace of $W^{1, p}(\Omega)$, defined by

$$
H=\left\{u \in W^{1, p}(\Omega): \int_{\Omega}\left(h(x)|\nabla u|^{p}+b(x)|u|^{p}\right) d x<+\infty\right\}
$$

and $H$ can be endowed with the norm

$$
\|u\|_{H}=\left(\int_{\Omega} h(x)|\nabla u|^{p}+b(x)|u|^{p} d x\right)^{\frac{1}{p}} .
$$

Applying the method as those used in [14] or [5], we can prove that:

Proposition 1.1. $H$ is a Banach space. The embedding continuous $H \hookrightarrow$ $W^{1, p}(\Omega)$ holds true.

Proof. It is clear that $H$ is a normed space. Let $\left\{u_{m}\right\}$ be a Cauchy sequence in $H$. Then

$$
\lim _{m, k \rightarrow \infty} \int_{\Omega}\left(h(x)\left|\nabla\left(u_{m}-u_{k}\right)\right|^{p}+b(x)\left|u_{m}-u_{k}\right|^{p}\right) d x=0
$$

and $\left\{\left\|u_{m}\right\|_{H}\right\}$ is bounded. 
Since $\left\|u_{m}-u_{k}\right\|_{W^{1, p}(\Omega)} \leq \bar{b}\left\|u_{m}-u_{k}\right\|_{H}, \bar{b}$ is a positive constant for all $m, k$, $\left\{u_{m}\right\}$ is also a Cauchy sequence in $W^{1, p}(\Omega)$ and it converges to $u$ in $W^{1, p}(\Omega)$, i.e.,

$$
\lim _{m \rightarrow+\infty} \int_{\Omega}\left(\left|\nabla u_{m}-\nabla u\right|^{p}+\left|u_{m}-u\right|^{p}\right) d x=0
$$

It follows the sequence $\left\{\nabla u_{m}\right\}$ converges to $\nabla u$ and $\left\{u_{m}\right\}$ converges to $u$ in $L^{p}(\Omega)$. Therefore $\left\{\nabla u_{m}(x)\right\}$ converges to $\nabla u(x)$ and $\left\{u_{m}(x)\right\}$ converges to $\{u(x)\}$ for almost everywhere $x \in \Omega$. Applying Fatou's lemma we get

$\int_{\Omega}\left(h(x)|\nabla u|^{p}+b(x)|u|^{p}\right) d x \leq \lim _{m \rightarrow+\infty} \inf \int_{\Omega}\left(h(x)\left|\nabla u_{m}\right|^{p}+b(x)\left|u_{m}\right|^{p}\right) d x<+\infty$.

Hence $u \in H$. Applying again Fatou's lemma

$$
\begin{aligned}
0 & \leq \lim _{m \rightarrow+\infty} \int_{\Omega}\left(h(x)\left|\nabla u_{m}-\nabla u\right|^{p}+b(x)\left|u_{m}-u\right|^{p}\right) d x \\
& \leq \lim _{m \rightarrow+\infty}\left[\lim _{k \rightarrow+\infty} \inf \int_{\Omega}\left(h(x)\left|\nabla u_{m}-\nabla u_{k}\right|^{p}+b(x)\left|u_{m}-u_{k}\right|^{p}\right) d x\right]=0 .
\end{aligned}
$$

Hence $\left\{u_{m}\right\}$ converges to $u$ in $H$. Thus $H$ is a Banach space and the continuous embedding $H \hookrightarrow W^{1, p}(\Omega)$ holds true.

Definition 1.1. A function $u \in H$ is a weak solution of the problem (1.1) if and only if

$$
\int_{\Omega} h(x)|\nabla u|^{p-2} \nabla u \nabla \varphi d x+\int_{\Omega} b(x)|u|^{p-2} u \varphi d x-\int_{\Omega} f(x, u) \varphi d x=0
$$

for all $\varphi \in C_{0}^{\infty}(\bar{\Omega})$.

Remark 1.1. If $u_{0} \in C_{0}^{\infty}(\bar{\Omega})$ satisfied the condition (1.2), hence $u_{0}$ is a classical solution of the problem (1.1). Indeed, since $u_{0} \in C_{0}^{\infty}(\bar{\Omega})$, supp $u_{0}$ compact, hence there exists $R>0$ large enough such that $\partial \Omega \subset B_{R}(0)$, supp $u_{0} \subset$ $\bar{\Omega} \cap B_{R}(0)$ where $B_{R}(0)$ is ball of radius $R$.

By denote $\Omega_{R}=\Omega \cap B_{R}(0)$, then from (F1) we have

$$
\int_{\Omega_{R}} h(x)\left|\nabla u_{0}\right|^{p-2} \nabla u_{0} \nabla \varphi d x+\int_{\Omega_{R}} b(x)\left|u_{0}\right|^{p-2} u_{0} \varphi d x-\int_{\Omega_{R}} f\left(x, u_{0}\right) \varphi d x=0
$$

for all $\varphi \in C_{0}^{\infty}(\bar{\Omega})$.

Applying Green's formula and remark that supp $u_{0} \subset \bar{\Omega} \cap B_{R}(0)$ we get

$$
\begin{aligned}
& \left.\int_{\Omega_{R}}-\operatorname{div}\left(h(x)\left|\nabla u_{0}\right|^{p-2} \nabla u_{0}\right) \varphi+b(x)\left|u_{0}\right|^{p-2} u_{0} \varphi\right) d x \\
& +\int_{\partial \Omega} h(x)\left|\nabla u_{0}\right|^{p-2} \frac{\partial u_{0}}{\partial n} \varphi d \sigma-\int_{\Omega_{R}} f\left(x, u_{0}\right) \varphi d x=0 \text { for all } \varphi \in C_{0}^{\infty}(\bar{\Omega}) .
\end{aligned}
$$

This implies that

$$
\int_{\Omega_{R}}\left(-\operatorname{div}\left(h(x)\left|\nabla u_{0}\right|^{p-2} \nabla u_{0}\right) \varphi+b(x)\left|u_{0}\right|^{p-2} u_{0} \varphi\right) d x-\int_{\Omega_{R}} f\left(x, u_{0}\right) \varphi d x=0
$$


for all $\varphi \in C_{0}^{\infty}\left(\Omega_{R}\right)$. From this it follows that

$$
\left\{\begin{array}{l}
-\operatorname{div}\left(h(x)\left|\nabla u_{0}\right|^{p-2} \nabla u_{0}\right)+b(x)\left|u_{0}\right|^{p-2} u_{0}=f\left(x, u_{0}\right) \text { in } \Omega, \\
\frac{\partial u_{0}}{\partial n}=0 \text { on } \partial \Omega .
\end{array}\right.
$$

Thus $u_{0}$ is a classical solution of (1.1).

Our main result given by the following theorem:

Theorem 1.1. Assuming hypotheses (F1)-(F3) are fulfilled then the problem (1.1) has at least one nontrivial weak solution in $H$.

Theorem 1.1 will be proved by using a variation of the Mountain pass theorem in $[6]$.

\section{Existence of a weak solution}

We define the functional $J: H \longrightarrow \mathbb{R}$ by

$$
\begin{aligned}
J(u) & =\frac{1}{p} \int_{\Omega} h(x)|\nabla u|^{p} d x+\frac{1}{p} \int_{\Omega} b(x)|u|^{p} d x-\int_{\Omega} F(x, u) d x \\
& =T(u)-P(u),
\end{aligned}
$$

where

and

$$
T(u)=\frac{1}{p} \int_{\Omega} h(x)|\nabla u|^{p} d x+\frac{1}{p} \int_{\Omega} b(x)|u|^{p} d x
$$

$$
P(u)=\int_{\Omega} F(x, u) d x .
$$

Firstly we remark that, due to the presence of $h(x) \in L_{\text {loc }}^{1}(\bar{\Omega})$, in general, the functional $T$ does not belong to $C^{1}(H)$. This mean that we cannot apply the classical Mountain pass theorem by Ambrossetti-Rabinowitz. In order to overcome this difficulty, we shall apply a weak version of the Mountain pass theorem introduced by D. M. Duc ([6]). Now we first recall the following useful concept:

Definition 2.1. Let $J$ be a functional from a Banach space $Y$ into $\mathbb{R}$. We say that $J$ is weakly continuously differentiable on $Y$ if and only if three following conditions are satisfied:

(i) $J$ is continuous on $Y$.

(ii) For any $u \in Y$ there exists a linear map $D J(u)$ from $Y$ into $\mathbb{R}$ such that

$$
\lim _{t \rightarrow 0} \frac{J(u+t \varphi)-J(u)}{t}=\langle D J(u), \varphi\rangle, \forall \varphi \in Y .
$$

(iii) For any $\varphi \in Y$, the map $u \mapsto\langle D J(u), \varphi\rangle$ is continuous on $Y$.

Proposition 2.1. Assuming hypotheses of Theorem 1.1 are fulfilled. We assert that 
(i) P is continuous on $H$. Moreover, $P$ is weakly continuously differentiable on $H$ and

$$
\langle D P(u), v\rangle=\int_{\Omega} f(x, u) v d x, \quad \forall u, v \in H .
$$

(ii) $T$ is continuous on $H$.

(iii) $T$ is weakly continuously differentiable on $H$ and

$$
\langle D T(u), v\rangle=\int_{\Omega}\left(h(x)|\nabla u|^{p-2} \nabla u \nabla v+b(x)|u|^{p-2} u v\right) d x, \quad \forall u, v \in H .
$$

Thus $J=T-P$ is weakly continuously differentiable on $H$ and

$$
\begin{aligned}
& \langle D J(u), v\rangle=\int_{\Omega}\left(h(x)|\nabla u|^{p-2} \nabla u \nabla v+b(x)|u|^{p-2} u v\right) d x-\int_{\Omega} f(x, u) v d x \\
& \forall u, v \in H .
\end{aligned}
$$

Proof. (i) By hypotheses of Theorem 1.1, applying Theorem C1 in [11, p. 248], we have $P \in C^{1}\left(W^{1, p}(\Omega)\right)$. Since the embedding $H \hookrightarrow W^{1, p}(\Omega)$ is continuous, we also have $P \in C^{1}(H)$ and then $P$ is weakly continuously differentiable on H. Moreover,

$$
\langle D P(u), v\rangle=\int_{\Omega} f(x, u) v d x \quad \forall u, v \in H .
$$

(ii) Let $\left\{u_{m}\right\}$ be a sequence converging to $u$ in $H$, i.e.,

$$
\lim _{m \longrightarrow+\infty} \int_{\Omega}\left(h(x)\left|\nabla u_{m}-\nabla u\right|^{p}+b(x)\left|u_{m}-u\right|^{p}\right) d x=0 .
$$

Then $\left\{\left\|u_{m}\right\|_{H}\right\}$ is bounded.

First we observe that: for some $\theta \in(0,1)$ :

$$
\begin{aligned}
\left.|| \nabla u_{m}\right|^{p}-|\nabla u|^{p} \mid & =p\left|\nabla u_{m}+\theta\left(\nabla u_{m}-\nabla u\right)\right|^{p-1}\left|\nabla u_{m}-\nabla u\right| \\
& \leq p 2^{p-2}\left(\left|\nabla u_{m}\right|^{p-1}\left|\nabla u_{m}-\nabla u\right|+\left|\nabla u_{m}-\nabla u\right|^{p}\right) .
\end{aligned}
$$

Hence by applying the Holder's inequality we get

$$
\begin{aligned}
& \left.\left|\frac{1}{p} \int_{\Omega} h(x)\right| \nabla u_{m}\right|^{p} d x-\frac{1}{p} h(x)|\nabla u|^{p} d x \mid \\
\leq & \left.\frac{1}{p} \int_{\Omega} h(x)|| \nabla u_{m}\right|^{p}-|\nabla u|^{p} \mid d x \\
\leq & 2^{p-2} \int_{\Omega} h(x)\left|\nabla u_{m}\right|^{p-1}\left|\nabla u_{m}-\nabla u\right| d x+2^{p-2} \int_{\Omega} h(x)\left|\nabla u_{m}-\nabla u\right|^{p} d x \\
\leq & 2^{p-2}\left(\int_{\Omega}\left(h(x)^{\frac{p-1}{p}}\left|\nabla u_{m}\right|^{p-1}\right)^{\frac{p}{p-1}} d x\right)^{\frac{p-1}{p}}\left(\int_{\Omega}\left(h(x)\left|\nabla\left(u_{m}-u\right)\right|^{p}\right) d x\right)^{\frac{1}{p}} \\
& +2^{p-2} \int_{\Omega}\left(h(x)\left|\nabla\left(u_{m}-u\right)\right|^{p}\right) d x \\
\leq & c_{1}\left(\left\|u_{m}\right\|_{H}^{p-1}|| u_{m}-u\left\|_{H}+\right\| u_{m}-u \|_{H}^{p}\right) .
\end{aligned}
$$


Similarly, we also have

$$
\begin{aligned}
& \left.\left|\frac{1}{p} \int_{\Omega} b(x)\right| u_{m}\right|^{p} d x-\frac{1}{p} \int_{\Omega} b(x)|u|^{p} d x \mid \\
\leq & c_{2}\left(\left\|u_{m}\right\|_{H}^{p-1}\left\|u_{m}-u\right\|_{H}+\left\|u_{m}-u\right\|_{H}^{p}\right) .
\end{aligned}
$$

Combining (2.6) and (2.7) we have

$$
\left|T\left(u_{m}\right)-T(u)\right| \leq c_{3}\left(\left\|u_{m}\right\|_{H}^{p-1}\left\|u_{m}-u\right\|_{H}+\left\|u_{m}-u\right\|_{H}^{p}\right)
$$

with $c_{1}, c_{2}, c_{3}>0$. Letting $m \rightarrow+\infty$ since $\left\|u_{m}-u\right\|_{H} \rightarrow 0$ and $\left\{\left\|u_{m}\right\|_{H}\right\}$ bounded, we obtain

$$
\lim _{m \rightarrow+\infty} T\left(u_{m}\right)=T(u) .
$$

Thus $T$ is continuous on $H$.

(iii) For all $u, v \in H$, any $t \in(-1,1) \backslash\{0\}$ and a.e. $x \in \Omega$ we have

$$
\begin{aligned}
& \left|\frac{h(x)|\nabla u+t \nabla v|^{p}-h(x)|\nabla u|^{p}}{t}\right| \\
= & p\left|\int_{0}^{1} h(x)\right| \nabla u+\left.s t \nabla v\right|^{p-2}(\nabla u+s t \nabla v) \nabla v d s \mid \\
\leq & p \int_{0}^{1} h(x)|\nabla u+s t \nabla v|^{p-1}|\nabla v| d s \leq p 2^{p-2} h(x)\left(|\nabla u|^{p-1}|\nabla v|+|\nabla v|^{p}\right) \\
\leq & p 2^{p-2}\left(h(x)^{\frac{p-1}{p}}|\nabla u|^{p-1} h(x)^{\frac{1}{p}}|\nabla v|+h(x)|\nabla v|^{p}\right) .
\end{aligned}
$$

Since $u, v \in H$, we observe that

$$
\begin{aligned}
& \int_{\Omega}\left(h(x)^{\frac{p-1}{p}}|\nabla u|^{p-1} h(x)^{\frac{1}{p}}|\nabla v|+h(x)|\nabla v|^{p}\right) d x \\
\leq & \left(\int_{\Omega}\left(h(x)^{\frac{p-1}{p}}|\nabla u|^{p-1}\right)^{\frac{p}{p-1}} d x\right)^{\frac{p-1}{p}}\left(\int_{\Omega} h(x)|\nabla v|^{p} d x\right)^{\frac{1}{p}}+c_{5}\|v\|_{H}^{p} \\
\leq & c_{4}\|u\|_{H}^{p-1}\|v\|_{H}+c_{5}\|v\|_{H}^{p}<+\infty,
\end{aligned}
$$

where $c_{4}, c_{5}$ two positive constants.

Hence $G(x)=h(x)|\nabla u|^{p-1}|\nabla v|+h(x)|\nabla v|^{p} \in L^{1}(\Omega)$. Applying the Lebesgue dominated convergence theorem we get

$$
\lim _{t \rightarrow 0} \int_{\Omega} \frac{h(x)|\nabla u+t \nabla v|^{p}-h(x)|\nabla u|^{p}}{t} d x=p \int_{\Omega} h(x)|\nabla u|^{p-2} \nabla u \nabla v d x .
$$

Similarly we also have

$$
\lim _{t \rightarrow 0} \int_{\Omega} \frac{b(x)|u+t v|^{p}-b(x)|u|^{p}}{t} d x=p \int_{\Omega} b(x)|u|^{p-2} u v d x .
$$

This implies that

$$
\langle D T(u), v\rangle=\lim _{t \rightarrow 0} \frac{T(u+t v)-T(u)}{t}=\int_{\Omega}\left(h(x)|\nabla u|^{p-2} \nabla u \nabla v+b(x)|u|^{p-2} u v\right) d x .
$$


Thus $T$ is weakly differentiable on $H$.

Let $v \in H$ be fixed, we now prove that the map $u \mapsto\langle D T(u), v\rangle$ is continuous on $H$.

Assume $u_{m} \rightarrow u$ in $H$, that is

$$
\lim _{m \rightarrow+\infty} \int_{\Omega}\left(h(x)\left|\nabla u_{m}-\nabla u\right|^{p}+b(x)\left|u_{m}-u\right|^{p}\right) d x=0 .
$$

By hypotheses (H) and (B) it follows that $\nabla u_{m} \rightarrow \nabla u$ and $u_{m} \rightarrow u$ in $L^{p}(\Omega)$. Applying Theorem C.2 in [11, p. 249] for function $g(x, s)=|s|^{p-2} s$, we deduce that

$$
g\left(x, \nabla u_{m}\right)=\left|\nabla u_{m}\right|^{p-2} \nabla u_{m} \longrightarrow|\nabla u|^{p-2} \nabla u
$$

and

$$
g\left(x, u_{m}\right)=\left|u_{m}\right|^{p-2} u_{m} \longrightarrow|u|^{p-2} u
$$

in $\left(L^{\frac{p}{p-1}}(\Omega)\right)^{N}$ as $m \rightarrow+\infty$, where $\left(L^{r}(\Omega)\right)^{N}=L^{r}(\Omega) \times L^{r}(\Omega) \times \cdots \times L^{r}(\Omega)$ ( $N$ times). Using this fact we shall proved that the map $u \rightarrow\langle D T(u), v\rangle$ is continuous on $H$ for every $v$ fixed in $H$.

Indeed for $\varphi \in C_{0}^{\infty}(\bar{\Omega}), \omega=\operatorname{supp} \varphi$, we have

$$
\begin{aligned}
& \left|\left\langle D T\left(u_{m}\right)-D T(u), \varphi\right\rangle\right| \\
= & \left|\int_{\Omega}\left\{h(x)\left(\left|\nabla u_{m}\right|^{p-2} \nabla u_{m}-|\nabla u|^{p-2} \nabla u\right) \nabla \varphi+b(x)\left(\left|u_{m}\right|^{p-2} u_{m}-|u|^{p-2} u\right) \varphi\right\} d x\right| \\
= & \left|\int_{\omega}\left\{h(x)\left(\left|\nabla u_{m}\right|^{p-2} \nabla u_{m}-|\nabla u|^{p-2} \nabla u\right) \nabla \varphi+b(x)\left(\left|u_{m}\right|^{p-2} u_{m}-|u|^{p-2} u\right) \varphi\right\} d x\right| \\
\leq & C(\varphi)\left\{\left\|g\left(x, \nabla u_{m}\right)-g(x, \nabla u)\right\|_{L^{\frac{p}{p-1}}(\omega)}|| \nabla \varphi \|_{L^{p}(\omega)}\right. \\
& \left.+\left\|g\left(x, u_{m}\right)-g(x, u)\right\|_{L^{\frac{p}{p-1}}(\omega)}\|\varphi\|_{L^{p}(\omega)}\right\},
\end{aligned}
$$

where $C(\varphi)$ is a constant positive. From this letting $m \rightarrow+\infty$ we get

$$
\lim _{m \rightarrow+\infty}\left|\left\langle D T\left(u_{m}\right)-D T(u), \varphi\right\rangle\right|=0 .
$$

Since $C_{0}^{\infty}(\bar{\Omega})$ is dense in $H$ we deduce that for every $v \in H$ fixed

$$
\lim _{m \rightarrow+\infty}\left|\left\langle D T\left(u_{m}\right)-D T(u), v\right\rangle\right|=0 \text {. }
$$

The proof of Proposition 2.1 is complete.

Proposition 2.2. Suppose that sequence $\left\{u_{m}\right\}$ is weakly converging to $u$ in $W^{1, p}(\Omega)$. Then we have

$$
T(u) \leq \lim _{m \rightarrow+\infty} \inf T\left(u_{m}\right)
$$

Proof. Since $\left\{u_{m}\right\}$ weakly converging in $W^{1, p}(\Omega)$ hence for all bounded $\Omega^{\prime} \subset \subset$ $\Omega,\left\{u_{m}\right\}$ is also weakly converging in $W^{1, p}\left(\Omega^{\prime}\right)$. By compactness of the embedding $W^{1, p}\left(\Omega^{\prime}\right)$ into $L^{p}\left(\Omega^{\prime}\right)$, the sequence $\left\{u_{m}\right\}$ converges strongly in $L^{p}\left(\Omega^{\prime}\right)$ 
then $\left\{u_{m}\right\}$ converges strongly in $L^{1}\left(\Omega^{\prime}\right)$. Applying Theorem 1.6 in [6, p. 9] or Theorem 4.5 [8, p. 129], we deduce that

$$
T(u) \leq \lim _{m \rightarrow+\infty} \inf T\left(u_{m}\right)
$$

The proof of Proposition 2.2 is complete.

Proposition 2.3. The functional $J: H \longrightarrow \mathbb{R}$ is defined by (2.4), i.e.,

$$
J(u)=T(u)-P(u), \quad u \in H
$$

satisfies the Palais-Smale condition on $H$.

Proof. Let $\left\{u_{m}\right\}$ be a sequence in $H$ such that

$$
\lim _{m \rightarrow \infty} J\left(u_{m}\right)=c, \quad \lim _{m \rightarrow+\infty}\left\|D J\left(u_{m}\right)\right\|_{H^{*}}=0 .
$$

First, we shall proved that $\left\{u_{m}\right\}$ is bounded in $H$. We suppose by contradiction that $\left\{u_{m}\right\}$ is not bounded in $H$. Then there exists a subsequence $\left\{u_{m_{k}}\right\}$ of $\left\{u_{m}\right\}$ such that $\left\|u_{m_{k}}\right\|_{H} \rightarrow+\infty$ as $k \rightarrow+\infty$. Observe further that

$$
\begin{aligned}
& J\left(u_{m_{k}}\right)-\frac{1}{\mu}\left\langle D J\left(u_{m_{k}}\right), u_{m_{k}}\right\rangle \\
= & T\left(u_{m_{k}}\right)-\frac{1}{\mu}\left\langle D T\left(u_{m_{k}}\right), u_{m_{k}}\right\rangle+\frac{1}{\mu}\left\langle D P\left(u_{m_{k}}\right), u_{m_{k}}\right\rangle-P\left(u_{m_{k}}\right) \\
\geq & \left(\frac{1}{p}-\frac{1}{\mu}\right)\left\|u_{m_{k}}\right\|_{H}^{p}
\end{aligned}
$$

yields

$$
\begin{aligned}
J\left(u_{m_{k}}\right) & \geq\left(\frac{1}{p}-\frac{1}{\mu}\right)\left\|u_{m_{k}}\right\|_{H}^{p}+\frac{1}{\mu}\left\langle D J\left(u_{m_{k}}\right), u_{m_{k}}\right\rangle \\
& \geq\left(\frac{1}{p}-\frac{1}{\mu}\right)\left\|u_{m_{k}}\right\|_{H}^{p}-\frac{1}{\mu}\left\|D J\left(u_{u_{m_{k}}}\right)\right\|_{H^{*}}\left\|u_{m_{k}}\right\|_{H} \\
& \geq\left\|u_{m_{k}}\right\|_{H}\left(\gamma_{0}\left\|u_{m_{k}}\right\|_{H}^{p-1}-\frac{1}{\mu}\left\|D J\left(u_{m_{k}}\right)\right\|_{H^{*}}\right),
\end{aligned}
$$

where $\gamma_{0}=\frac{1}{p}-\frac{1}{\mu}>0$.

From this letting $k \rightarrow+\infty$, since $\left\|u_{m_{k}}\right\|_{H} \rightarrow+\infty,\left\|D J\left(u_{m_{k}}\right)\right\|_{H^{*}} \rightarrow 0$, we deduce $J\left(u_{m_{k}}\right) \rightarrow+\infty$ yields a contradiction. Hence $\left\{u_{m}\right\}$ is bounded in $H$. By the continuous embedding $H$ into $W^{1, p}(\Omega),\left\{u_{m}\right\}$ is bounded in $W^{1, p}(\Omega)$. Therefore, there exists a subsequence $\left\{u_{m_{k}}\right\}$ of $\left\{u_{m}\right\}$ converging weakly to $u$ in $W^{1, p}(\Omega)$. Since the embedding $W^{1, p}(\Omega) \hookrightarrow L^{p^{*}}(\Omega)$ is continuous, the subsequence $\left\{u_{m_{k}}\right\}$ converges weakly to $u$ in $L^{p^{*}}(\Omega)$ and $u_{m_{k}} \rightarrow u$ a.e. $x \in \Omega$. It follows that $\left\{u_{m_{k}}\right\}$ is bounded in $L^{p^{*}}(\Omega)$, that is there exists a constant $M>0$ such that

$$
\left\|u_{m_{k}}\right\|_{L^{p^{*}}(\Omega)} \leq M \text { for all } k=1,2, \ldots
$$


We remark that by hypotheses (F2) and (F3) we get

$$
0 \leq F(x, z) \leq \tau(x)|z|^{r+1} \text { for } x \in \bar{\Omega}, z \in \mathbb{R}-\{0\},
$$

where $\tau(x) \in L^{r_{0}}(\Omega) \cap L^{\infty}(\Omega)$.

Then by Holder's inequality and remark that $\frac{1}{r_{0}}+\frac{r+1}{p^{*}}=1$ we deduce

$$
\begin{aligned}
P\left(u_{m_{k}}\right)=\int_{\Omega} F\left(x, u_{m_{k}}\right) d x & \leq \int_{\Omega} \tau(x)\left|u_{m_{k}}\right|^{r+1} \\
& \leq\|\tau(x)\|_{L^{r_{0}(\Omega)}}\left\|u_{m_{k}}\right\|_{L^{p^{*}}(\Omega)}^{r+1} \\
& \leq M^{r+1}\|\tau(x)\|_{L^{r_{0}(\Omega)}} .
\end{aligned}
$$

By Proposition 2.2 we get

$$
\begin{aligned}
T(u) & \leq \lim _{k \rightarrow+\infty} \inf T\left(u_{m_{k}}\right) \leq \lim _{k \rightarrow+\infty}\left[P\left(u_{m_{k}}\right)+J\left(u_{m_{k}}\right)\right] \\
& \leq c+\|\tau(x)\|_{L^{r_{0}}(\Omega)} M^{r+1}<+\infty
\end{aligned}
$$

Thus $u \in H$.

Since $\left\{u_{m_{k}}\right\}$ is weakly converges to $u$ in $L^{p^{*}}(\Omega)$ and $u_{m_{k}} \rightarrow u$ a.e. $x \in \Omega$. Then it is clear that $\left|u_{m_{k}}\right|^{r-1} u_{m_{k}}$ is converges weakly to $|u|^{r-1} u$ in $L^{\frac{p^{*}}{r}}(\Omega)$. With similar arguments as those in [9], we define the map $K(u): L^{\frac{p^{*}}{r}}(\Omega) \longrightarrow \mathbb{R}$ by

$$
\langle K(u), \omega\rangle=\int_{\Omega} \tau(x) u \omega d x \quad \text { for } \omega \in L^{\frac{p^{*}}{r}}(\Omega) .
$$

We remark that $K(u)$ is linear and continuous provided that $\tau(x) \in L^{r_{0}}(\Omega)$, $u \in L^{p^{*}}(\Omega), \omega \in L^{\frac{p^{*}}{r}}(\Omega)$ and $\frac{1}{r_{0}}+\frac{1}{p^{*}}+\frac{r}{p^{*}}=1$. Hence

$$
\left\langle K(u),\left|u_{m_{k}}\right|^{r-1} u_{m_{k}}\right\rangle \longrightarrow\left\langle K(u),|u|^{r-1} u\right\rangle \text { as } k \rightarrow+\infty,
$$

i.e.,

$$
\lim _{k \rightarrow+\infty} \int_{\Omega} \tau(x)\left|u_{m_{k}}\right|^{r-1} u_{m_{k}} u d x=\int_{\Omega} \tau(x)|u|^{r+1} d x .
$$

Similarly we also have

$$
\lim _{k \rightarrow+\infty} \int_{\Omega} \tau(x)\left|u_{m_{k}}\right|^{r+1} d x=\int_{\Omega} \tau(x)|u|^{r+1} d x .
$$

Combining (2.8), (2.9) we get

$$
\lim _{k \rightarrow+\infty} \int_{\Omega} \tau(x)\left|u_{m_{k}}\right|^{r-1} u_{m_{k}}\left(u_{m_{k}}-u\right) d x=0 .
$$

By (2.10), (F1), (F2) we obtain

$$
\lim _{m \rightarrow+\infty} \int_{\Omega} f\left(x, u_{m_{k}}\right)\left(u_{m_{k}}-u\right) d x=0,
$$

i.e.,

$$
\lim _{k \rightarrow+\infty}\left\langle D P\left(u_{m_{k}}\right), u_{m_{k}}-u\right\rangle=0 .
$$


It follows from (2.11) that

$$
\begin{aligned}
\lim _{k \rightarrow+\infty}\left\langle D T\left(u_{m_{k}}\right), u_{m_{k}}-u\right\rangle= & \lim _{k \rightarrow+\infty}\left\langle D J\left(u_{m_{k}}\right),\left(u_{m_{k}}-u\right)\right\rangle \\
& +\lim _{k \rightarrow+\infty}\left\langle D P\left(u_{m_{k}}\right),\left(u_{m_{k}}-u\right)\right\rangle=0 .
\end{aligned}
$$

Moreover, since $T$ is convex we have

$$
T(u)-T\left(u_{m_{k}}\right) \geq\left\langle D T\left(u_{m_{k}}, u-u_{m_{k}}\right)\right\rangle .
$$

Letting $k \rightarrow+\infty$ we obtain that

$$
\begin{aligned}
T(u)-\lim _{k \rightarrow+\infty} T\left(u_{m_{k}}\right) & =\lim _{k \rightarrow+\infty}\left[T(u)-T\left(u_{m_{k}}\right)\right] \\
& \geq \lim _{k \rightarrow+\infty}\left\langle D T\left(u_{m_{k}}\right), u-u_{m_{k}}\right\rangle=0 .
\end{aligned}
$$

Thus

$$
T(u) \geq \lim _{k \rightarrow+\infty} T\left(u_{m_{k}}\right) .
$$

On other hand, by Proposition 2.2 we have

$$
T(u) \leq \lim _{k \rightarrow+\infty} \inf T\left(u_{m_{k}}\right) .
$$

Hence, from two above inequalities, we get $T(u)=\lim _{k \rightarrow+\infty} T\left(u_{m_{k}}\right)$.

Now, we shall prove that the subsequence $\left\{u_{m_{k}}\right\}$ converges strongly to $u$ in $H$, i.e., $\lim _{k \rightarrow+\infty}\left\|u_{m_{k}}-u\right\|_{H}=0$.

Indeed, we suppose by contradiction that $\left\{u_{m_{k}}\right\}$ does not converge strongly to $u$ in $H$. Then there exist a constant $\varepsilon_{0}>0$ and a subsequence $\left\{u_{m_{k_{j}}}\right\}$ of $\left\{u_{m_{k}}\right\}$ such that $\left\|u_{m_{k_{j}}}-u\right\|_{H} \geq \varepsilon_{0}$ for any $j=1,2, \ldots$

By recalling the Clarkson's inequality

$$
\left|\frac{\alpha+\beta}{2}\right|^{p}+\left|\frac{\alpha-\beta}{2}\right|^{p} \leq \frac{1}{2}\left(|\alpha|^{p}+|\beta|^{p}\right), \forall \alpha, \beta \in \mathbb{R} .
$$

We deduce that

$$
\frac{1}{2} T(u)+\frac{1}{2} T(v)-T\left(\frac{u+v}{2}\right) \geq T\left(\frac{u-v}{2}\right), \forall u, v \in H .
$$

From this, for any $j=1,2, \ldots$, we have

$$
\frac{1}{2} T\left(u_{m_{k_{j}}}\right)+\frac{1}{2} T(u)-T\left(\frac{u_{m_{k_{j}}}+u}{2}\right) \geq T\left(\frac{u_{m_{k_{j}}}-u}{2}\right) .
$$

Remark that

$$
T\left(\frac{u_{m_{k_{j}}}-u}{2}\right)=\frac{1}{p 2^{p}}\left\|u_{m_{k_{j}}}-u\right\|_{H}^{p} \geq \frac{1}{p 2^{p}} \varepsilon_{0}^{p} .
$$

We get

$$
\frac{1}{2} T\left(u_{m_{k_{j}}}\right)+\frac{1}{2} T(u)-T\left(\frac{u_{m_{k_{j}}}+u}{2}\right) \geq \frac{1}{p 2^{p}} \varepsilon_{0}^{p} .
$$


Again instead of the remark that since $\left\{\frac{u_{m_{k_{j}}}+u}{2}\right\}$ converges weakly to $u$ in $W^{1, p}(\Omega)$, by Proposition 2.2 we have

$$
T(u) \leq \lim _{j \rightarrow+\infty} \inf T\left(\frac{u_{m_{k_{j}}}+u}{2}\right) .
$$

From (2.12), letting $j \rightarrow+\infty$ we obtain that

$$
T(u)-\lim _{j \rightarrow+\infty} \inf T\left(\frac{u_{m_{k_{j}}}+u}{2}\right) \geq \frac{1}{p 2^{p}} \varepsilon_{0}^{p} .
$$

Hence $0 \geq \frac{1}{p 2^{p}} \varepsilon_{0}^{p}$, which is a contradiction.

Therefore, $\left\{u_{m_{k}}\right\}$ converges strongly to $u$ in $H$. Thus, the functional $J$ satisfies the Palais-Smale condition on $H$. The proof of Proposition 2.3 is complete.

We remark that the critical points of the functional $J$ correspond to the weak solutions of the problem (1.1). Thus our idea is to apply a variation of the Mountain pass theorem (see [6]) in order to obtain at least one non-trivial weak solution of the problem (1.1).

In what follows, we will prove proposition which shows that the functional $J$ has the Mountain pass geometry.

Proposition 2.4. (i) There exist $\alpha>0$ and $\rho>0$ such that $J(u) \geq \alpha>0$ for all $u \in H,\|u\|_{H}=\rho$.

(ii) There exists $u_{0} \in H,\left\|u_{0}\right\|_{H}>\rho$ and $J\left(u_{0}\right)<0$.

Proof. (i) Using (F2) and L'Hospistal theorem we have

$$
\lim _{z \rightarrow 0} \frac{F(x, z)}{z^{p}}=\lim _{z \rightarrow 0} \frac{f(x, z)}{p z^{p-1}}=\lim _{z \rightarrow 0} \frac{f_{z}^{\prime}(x, z)}{p(p-1) z^{p-2}}=0 .
$$

Thus

$$
\lim _{z \rightarrow 0} \frac{F(x, z)}{z^{p}}=0 .
$$

Using (F2) there exists $A$ a positive constant such that

$$
|f(x, z)| \leq A|z|^{r} .
$$

We integrate again

$$
0<F(x, z) \leq \bar{A}|z|^{r+1},
$$

where $\bar{A}$ is a positive constant. Then

$$
0 \leq \lim _{z \rightarrow+\infty} \frac{F(x, z)}{z^{\frac{N p}{N-p}}} \leq \lim _{z \rightarrow+\infty} \frac{\bar{A}|z|^{r+1}}{z^{\frac{N p}{N-p}}}=0
$$

with $r \in\left(p-1, \frac{N+p}{N-p}\right)$. Hence

$$
\lim _{z \rightarrow+\infty} \frac{F(x, z)}{z^{\frac{N p}{N-p}}}=0 .
$$


Using (2.13), (2.14), we obtain

$\forall \varepsilon>0, \exists \delta_{1}>0$ such that $\left|\frac{F(x, z)}{z^{p}}\right|<\varepsilon$ for all $z$ with $|z|<\delta_{1}$.

$\forall \varepsilon>0, \exists \delta_{2}>0$ such that $\left|\frac{F(x, z)}{z^{N-p}}\right|<\varepsilon$ for all $z$ with $|z|>\delta_{2}$.

Thus $\forall \varepsilon>0$, there exist $\delta_{1}, \delta_{2}>0$ such that

$$
F(x, z)<\varepsilon|z|^{p}, \quad|z|<\delta_{1} \text { and } F(x, z)<\varepsilon|z|^{\frac{N p}{N-p}}, \quad|z|>\delta_{2} .
$$

Using the relation $0<F(x, z) \leq \bar{A}|z|^{r+1}$ there exists a constant $b>0$ such that $F(x, z) \leq b$ for all $|z| \in\left[\delta_{1}, \delta_{2}\right]$. We conclude that for all $\varepsilon>0$, there exists $b_{\varepsilon}>0$ such that

$$
F(x, z) \leq \varepsilon|z|^{p}+b_{\varepsilon}|z|^{\frac{N p}{N-p}}
$$

Using (2.15) we have

$$
\begin{aligned}
J(u) & =\frac{1}{p}\|u\|_{H}^{p}-\int_{\Omega} F(x, u) d x \\
& \geq \frac{1}{p}\|u\|_{H}^{p}-\varepsilon \int_{\Omega}|u|^{p} d x-b_{\varepsilon} \int_{\Omega}|u|^{\frac{N p}{N-p}} d x .
\end{aligned}
$$

For $p \leq q \leq \frac{N p}{N-p}, W^{1, p}(\Omega) \hookrightarrow L^{q}(\Omega)$ is continuous. So the embedding $H \hookrightarrow$ $L^{q}(\Omega)$ is continuous, $|u|_{L^{q}(\Omega)} \leq c|| u \|_{H}$. Thus we have

$$
\begin{gathered}
|u|_{L^{p}} \leq C_{1}\|u\|_{H} . \\
|u|_{L^{N-p}} \leq C_{2}\|u\|_{H} .
\end{gathered}
$$

Therefore

$$
\begin{aligned}
J(u) & \geq \frac{1}{p}\|u\|_{H}^{p}-\varepsilon C_{1}^{p}\|u\|_{H}^{p}-b_{\varepsilon} C_{2}^{\frac{N p}{N-p}}\|u\|_{H}^{\frac{N p}{N-p}} \\
& \geq\|u\|_{H}^{p}\left(\frac{1}{p}-\varepsilon C_{1}^{p}-b_{\varepsilon} C_{2}^{\frac{N p}{N-p}}\|u\|^{\frac{N p}{N-p}-p}\right) .
\end{aligned}
$$

Letting $\varepsilon \in\left(0, \frac{1}{p C_{1}^{p}}\right)$ and $\|u\|_{H}=\rho$ small enough such that

$$
\frac{1}{p}-\varepsilon C_{1}^{p}-b_{\varepsilon} C_{2}^{\frac{N p}{N-p}}\|u\|_{H}^{\frac{N p}{N-p}-p}>0
$$

we obtain

$$
J(u) \geq\left(\frac{1}{p}-\varepsilon C_{1}^{p}-b_{\varepsilon} C_{2}^{\frac{N p}{N-p}}\|u\|^{\frac{N p}{N-p}-p}\right) \rho^{p}=\alpha>0 .
$$

ii) Denote $h(t)=\frac{F(x, t z)}{t^{\mu}}$ for all $t>0$.

Then using (F3) we get

$$
h^{\prime}(t)=\frac{1}{t^{\mu+1}}[t z f(x, t z)-\mu F(x, t z)] \geq 0, \forall t>0 .
$$


Thus we deduce for any $t \geq 1, F(x, t z) \geq t^{\mu} F(x, z)$. Let $w_{0} \in C_{0}^{\infty}(\bar{\Omega})$ be such that meas $\left(\left\{x \in(\bar{\Omega}):\left|w_{0}(x)\right|>0\right\}\right)>1$ then with $t>1$ we get

$$
\begin{aligned}
J\left(t w_{0}\right) & =\int_{\Omega} \frac{1}{p}\left(h(x)\left|\nabla\left(t w_{0}\right)\right|^{p}+b(x)\left|t w_{0}\right|^{p}\right) d x-\int_{\Omega} F\left(x, t w_{0}\right) d x \\
& =\int_{\Omega} \frac{t^{p}}{p}\left(h(x)\left|\nabla w_{0}\right|^{p}+b(x)\left|w_{0}\right|^{p}\right) d x-\int_{\Omega} F\left(x, t w_{0}\right) d x \\
& \leq \frac{t^{p}}{p}\left\|w_{0}\right\|_{H}^{p}-t^{\mu} \int_{\Omega} F\left(x, w_{0}\right) d x .
\end{aligned}
$$

Since $\mu>p$, the right hand-side of above inequality converges to $-\infty$ when $t \rightarrow+\infty$. Then there exists $t_{0}>0$ such that $\left\|t_{0} w_{0}\right\|_{H}>\rho$ and $J\left(t_{0} w_{0}\right)<0$. Set $u_{0}=t_{0} w_{0}$, we have $J\left(u_{0}\right)<0$ and $\left\|u_{0}\right\|>\rho$.

The proof of Proposition 2.4 is complete.

Proposition 2.5. (i) $J(0)=0$.

(ii) The acceptable set $G=\left\{\gamma \in C([0,1], H): \gamma(0)=0, \gamma(1)=u_{0}\right\}$ is not empty, where $u_{0}$ is given in Proposition 2.4.

It is clear that: (i) follows from (F1) and the definition of $J$.

(ii) Let $\gamma(t)=t u_{0}$, then $\gamma(t) \in G$.

Proof of Theorem 1.1. By Propositions 2.1-2.5, all assumptions of the variations of the Mountain pass theorem introduced in [6] are satisfied. Therefore there exists $\hat{\mathrm{u}} \in H$ such that

$$
0<\alpha \leq J(\hat{\mathrm{u}})=\inf \{\max J(\gamma([0,1])): \gamma \in G\}
$$

and $\langle D J(\hat{\mathrm{u}}), v\rangle=0$ for all $v \in H$, i.e., $\hat{\mathrm{u}}$ is a weak solution of the problem (1.1). Moreover since $J(\hat{\mathrm{u}})>0=J(0), \hat{\mathrm{u}}$ is a nontrivial weak solution of the problem (1.1). The Theorem 1.1 is completely proved.

\section{References}

[1] M. Alif and P. Omari, On a p-Neumann problem with asymptotically asymmetric perturbations, Nonlinear Anal. 51 (2002), no. 3, 369-389.

[2] G. Anello, Existence of infinitely many weak solutions for a Neumann problem, Nonlinear Anal. 57 (2004), no. 2, 199-209.

[3] P. A. Binding, P. Drábek, and Y. X. Huang, Existence of multiple solutions of critical quasilinear elliptic Neumann problems, Nonlinear Anal. 42 (2000), no. 4, 613-629.

[4] G. Bonanno and P. Candito, Three solutions to a Neumann problem for elliptic equations involving the p-Laplacian, Arch. Math. (Basel) 80 (2003), no. 4, 424-429.

[5] N. T. Chung and H. Q. Toan, Existence results for uniformly degenerate semilinear elliptic systems in $\mathbb{R}^{N}$, Glassgow Mathematical Journal 51 (2009), 561-570.

[6] D. M. Duc, Nonlinear singular elliptic equations, J. London Math. Soc. (2) 40 (1989), no. 3, 420-440.

[7] D. M. Duc and N. T. Vu, Nonuniformly elliptic equations of p-Laplacian type, Nonlinear Anal. 61 (2005), no. 8, 1483-1495.

[8] E. Giusti, Direct Methods in the Calculus of Variation World Scientific, New Jersey, 2003. 
[9] M. Mihăilescu, Existence and multiplicity of weak solution for a class of degenerate nonlinear elliptic equations, Boundary Value Problems 2006 (2006), Art ID 41295, $17 \mathrm{pp}$.

[10] B. Ricceri, Infinitely many solutions of the Neumann problem for elliptic equations involving the p-Laplacian, Bull. London Math. Soc. 33 (2001), no. 3, 331-340.

[11] M. Struwe, Variational Methods, Second Edition, Springer-Verlag, 2000.

[12] C. L. Tang, Solvability of Neumann problem for elliptic equations at resonance, Nonlinear Anal. 44 (2001), no. 3, 323-335.

[13] _ Some existence theorems for the sublinear Neumann boundary value problem, Nonlinear Anal. 48 (2002), no. 7, 1003-1011.

[14] H. Q. Toan and N. T. Chung, Existence of weak solutions for a class of nonuniformly nonlinear elliptic equations in unbounded domains, Nonlinear Anal. 70 (2009), no. 11, 3987-3996.

[15] H. Q. Toan and Q. A. Ngo, Multiplicity of weak solutions for a class of nonuniformly elliptic equations of p-Laplacian type, Nonlinear Anal. 70 (2009), no. 4, 1536-1546.

[16] X. Wu and K.-K. Tan, On existence and multiplicity of solutions of Neumann boundary value problems for quasi-linear elliptic equations, Nonlinear Anal. 65 (2006), no. 7, $1334-1347$.

Trinh Thi Minh Hang

DEPARTMENT OF InFormatics

Hanoi University of Civil Engineering

55 Giai Phong, Hanoi Vietnam

E-mail address: quoctrung032007@yahoo.com

HOANG QUOC TOAN

Department of Mathematics

Hanoi University of SCIENCE

334 Nguyen Trai, Hanoi, Vietnam

E-mail address: hq_toan@yahoo.com 